\section{C.002 HOW TO INFORM POLICY AND DECISION MAKERS ABOUT EVIDENCE-BASED PREVENTION}

Matej Košir*, Sanela Talić. Institute For Research and Development 'Utrip', Borovnica, Slovenia

10.1136/injuryprev-2021-safety. 157

Advocacy skills are essential for the prevention scientists, researchers and practitioners to potentially influence policy and decision making processes for better prevention policies and practice. Those skills are important especially in those prevention areas where so-called 'unhealthy' industries are influential and/or (at least) want to be important stakeholders in the field (such as alcohol, tobacco and pharmaceutical companies). They are also important to advocate for evidencebased prevention policies (e.g. alcohol, tobacco or drug policy) and practice, which should be fully in line with minimum quality standards in prevention. There is a growing need globally for advocacy knowledge and skills in prevention science and practice. Some key challenges regarding the needs for advocacy and training (in purpose to increase knowledge and skills) will be presented and discussed by the author. Based on authors extensive experience, advocacy could increase competences and skills for advocacy, such as forging stronger relationships with policy and decision makers and other key stakeholders in the field, advocating for the use of research to inform policy and decision makers about evidence-based policies and practice, providing expert testimony, writing position papers, press releases and social media posts, increasing the visibility and recognition of evidence-based prevention and committing to ongoing advocacy. Scientists, researchers and practitioners need to become more relevant to policy and decision makers if we want to achieve better results in prevention science and practice at international, regional or local level.

\section{C.003 ARE WE DOING WHAT WORKS TO PREVENT UNINTENTIONAL INJURY IN AUSTRALIAN CHILDREN?}

${ }^{1}$ Amy Bestman*, ${ }^{1}$ Kate Hunter, ${ }^{1,2}$ Julie Brown, ${ }^{3}$ Kate Curtise, ${ }^{4}$ Kathleen Clapham. ${ }^{1}$ The George Institute for Global Health, Newtown, Australia; ${ }^{2}$ Neuroscience Research Australia (NeuRA), Randwick, Australia; ${ }^{3}$ University of Sydney, Sydney, Australia; ${ }^{4}$ University of Wollongong, Wollogong, Australia

\subsection{6/injuryprev-2021-safety. 158}

Context In Australia, injury is the leading cause of death for children aged over 1 year and the highest cause of hospitalisation. There is good evidence for effectiveness of many existing injury prevention programs. Despite this evidence, there has been limited change in rates for specific injury areas and populations. To explore the gaps in injury prevention work, we examined whether Australian policy was consistent with bestpractice injury prevention research.

Process This work consisted of two stages. The first stage synthesised the evidence for unintentional child injury prevention including what is known to work to reduce inequities related to injury. Using this evidence, a framework was developed that included best practice measures and principles for child injury prevention. The second stage involved applying the framework to Australian policy documents to identify the extent the documents were 1) consistent with the evidence base, and 2) addressed specific inequities.
Outcomes There is a gap in prioritising inequity, a lag in uptake of some effective interventions into policy and legislation and a need for policy documents to focus more on reducing the inequities related to unintentional child injury. While some documents stated priority populations, there is a gap in specific measures and commitment to resourcing for groups that experience an unequal burden of injury.

Learning Outcomes The current work identified room for improvement in aligning Australian policy documents with best-practice interventions. This presentation will generate discussion around the actions required to ensure Australian policy is aligned with the evidence base.

\section{C.004 USING CAPACITY STRENGTHENING TO ENABLE EFFECTIVE INJURY PREVENTION IN NEPAL}

${ }^{1}$ Julie Mytton*, ${ }^{2}$ Matthew Ellis, ${ }^{3}$ Sunil Manhandar, ${ }^{3}$ Sunil Kumar Joshi. ${ }^{1}$ University of The West of UK; ${ }^{2}$ University of Bristol, UK; ${ }^{3}$ Kathmandu Medical College, Kathmandu, Nepal

\subsection{6/injuryprev-2021-safety. 159}

Context Through a collaboration between the University of the West of England, Bristol and Kathmandu Medical College in 2017, we established the Nepal Injury Research Centre. An innovative capacity strengthening programme underpins our ethos and activities, and contributes to SDGs 17 (Capacity development) and 4 (Education).

Process Facilitated through training leads in Nepal and the UK, we provide training to eight multidisciplinary Nepali researchers with variable experience. Each leads a study/study component, thereby learning-by-doing, and has an experienced research supervisor. Researchers have an annual training needs assessment to inform support provision.

Analysis Year 2 training needs assessments reveal increasing confidence and ownership of individual training requirements. Three researchers have completed international masters level degrees with the taught component taken abroad but dissertation data collection in-country. Three have attended international short courses and two have participated in the WHO Mentor-VIP programme. Six governance/methodology workshops have taken place.

Outcomes Projects have been successfully delivered and all researchers have opportunities for oral presentations and preparing publications. The team includes Nepali nationals purely based in Kathmandu, researchers having placements overseas, and Nepali diaspora resident in the UK. This helps to dilute the traditional donor/recipient dichotomy and has facilitated a mutually supportive team. Identifying and securing $\mathrm{PhD}$ scholarship funding is our next challenge.

Learning Outcomes Team building can be enhanced through capacity strengthening programmes. Funding from the UK National Institute for Health Research explicitly facilitates capacity strengthening based on an established UK training model. Blurring of national boundaries is a desirable outcome of the programme.

\section{C.005 USING SOCIAL MEDIA FOR INJURY PREVENTION: THE EXPERIENCE OF THE SAFETEA CAMPAIGN}

${ }^{1}$ Alan Emond*, ${ }^{2}$ Laura Cowley, ${ }^{2}$ Alison Kemp. ${ }^{1}$ University of Bristol, Bristol, UK; ${ }^{2}$ Cardiff University, Cardiff, UK

10.1136/injuryprev-2021-safety. 160 